\title{
Cerebral Microbleed Causing an Acute Stroke-like Episode in a CADASIL Patient
}

\author{
Paolo Vitali, Dan Boghen, Nicole Daneault, Laurent Guillon-Létourneau, Alexandre Y. Poppe
}

doi:10.1017/cjn.2014.29

Can J Neurol Sci. 2014; 41: 661-663

Cerebral microbleeds (CMBs), visualized on gradient-recalled echo (GRE) $\mathrm{T}_{2}$ or susceptibility-weighted imaging magnetic resonance imaging (MRI) sequences, and thought to reflect minor leakage from fragile arterial micro-vessels, are classically considered to be clinically silent. They are frequent incidental findings in MRI scans of healthy subjects, especially in the elderly population and even more prevalent in patients with acute ischemic and hemorrhagic stroke. However, increasing evidence has called into question the asymptomatic nature of CMBs. In particular, they appear to be associated with an increased risk of incident ischemic and hemorrhagic strokes as well as recurrent strokes. ${ }^{1}$

Greater controversy surrounds the possibility that CMBs can cause acute stroke-like episodes. In fact, CMBs are often associated with a degree of surrounding tissue necrosis, ${ }^{2}$ however, in theory, the brain tissue damaged by an acutely developing CMB is too small to produce focal neurological symptoms. Nevertheless, a few case reports of acute focal deficits with negative diffusionweighted imaging (DWI) brain-MRI sequences seem to suggest that a strategically located CMB could, indeed, cause an acute stroke syndrome. ${ }^{3}$ These few published reports have invariably described patients with classical risk factors for small vessel disease.

\section{Case Report}

We report a case of a 50 year-old right-handed man with no known vascular risk factors presenting with sudden onset of severe headache, vertigo, nausea, photophobia and horizontal diplopia. His past medical history was remarkable for migraine with aura and some transient stroke-like episodes. His mother died in her early fifties after suffering several "strokes". Neuroophthalmological examination early after onset of symptoms revealed left hypertropia, clockwise torsion of the eyes, right head tilt, counter-clockwise rotatory nystagmus and upbeat nystagmus in primary position. This was interpreted prior to the MRI as consistent with an ocular tilt reaction (OTR) possibly due to a lesion in the left rostral midbrain.

Investigations for a suspected posterior circulation stroke were carried out. Computed tomography angiography and serological investigations for stroke in the young adult were normal. Surprisingly, no acute ischemic lesion was observed on DWI sequences performed 24 hours post-onset, making an acute ischemic stroke very unlikely (DWI sequence parameters: 3 Tesla unit, Achieva X, Philips, the Netherlands, FOV $230 \times 230 \mathrm{~mm}$, b values: $0,500,1000$, slice thickness $5 \mathrm{~mm}$, interslice gap $1.0 \mathrm{~mm}$ ). However, the GRE sequence showed a pattern of multiple subcortical CMBs (GRE sequence parameters: TR/TE $500 / 23 \mathrm{~ms}$, FOV $230 \times 230 \mathrm{~mm}^{2}$, slice thickness $3 \mathrm{~mm}$, interslice gap $1 \mathrm{~mm}$ ).
In particular, one CMB was strategically located in the left rostral midbrain (Figure 1). Furthermore, brain MRI showed classical radiological findings of Cerebral Autosomal Dominant Arteriopathy with Subcortical Infarcts and Leukoencephalopathy (CADASIL) disease, including extensive leukoencephalopathy with preferential involvement of both temporal poles as well as several old lacunar infarcts (Figure 2). Notch3 mutation analysis identified a missense mutation in exon 4 of one of the two copies of the Notch3 gene (NOTCH3: c.421C>T; p.Arg141Cys) and confirmed the diagnosis of CADASIL.

The patient had completely recovered at clinical follow-up sixmonths later. Follow-up brain MRI (with high-resolution axial $\mathrm{T}_{2}$ TSE with $3 \mathrm{~mm}$ slice thickness and $0.1 \mathrm{~mm}$ interslice gap) performed at the same time did not show any lacunar infarct that could have been missed on the initial MRI and confirmed the persistence of the previously identified CMB. Given the presence of a CMB in a location corresponding to the clinical syndrome, we can reasonably argue that this radiological finding might be causally associated with his acute neurological presentation.

\section{DisCUSSION}

Although CMBs are not usually considered CADASILspecific radiological features, they are found in 31 to $69 \%$ of CADASIL patients, and are associated with an increased risk of intracerebral hemorrhage and cognitive decline, especially executive dysfunction. ${ }^{4}$ Nonetheless, the direct lesion-related effect of CMBs in stroke-like manifestations of CADASIL is still largely unknown. Our patient presented acute focal deficits consistent with a stroke of the posterior circulation, without evidence of a focal ischemic lesion on early post-onset brain MRI.

We acknowledge some potential limitations of our case report. First, we do not have an MRI scan preceding the clinical presentation to confirm that the CMB is acute. It is also possible that a very small lacunar infarction would have remained undetected using our conventional DWI sequence. High-resolution diffusion has been shown to increase the sensitivity for ischemic lesions, especially for cortical lesions. ${ }^{5}$ Diffusion-negative infarcts are more likely with lacunar infarction and posterior circulation lesions. ${ }^{6}$ In patients with persistent neurological deficits but a DWI-negative study, repeat MRI may show a delayed appearance of DWI lesions. ${ }^{7}$ However,

From the Department of Medicine (PV, DB, ND, AYP), Division of Neurology;

Department of Radiology (LG-L), Division of Neuroradiology, Hôpital Notre-Dame, Centre Hospitalier de l'Université de Montréal, Québec, Canada.

Received February 26, 2014. Final Revisions Submitted April 24, 2014 Correspondence to: Alexandre Y. Poppe, Hôpital Notre-Dame, Centre Hospitalier de l'Université de Montréal, Pavillon Deschamps1560, rue Sherbrooke est, Montréal, Québec H2L 4M1, Canada. Email: alexandre.yves.poppe@umontreal.ca. 


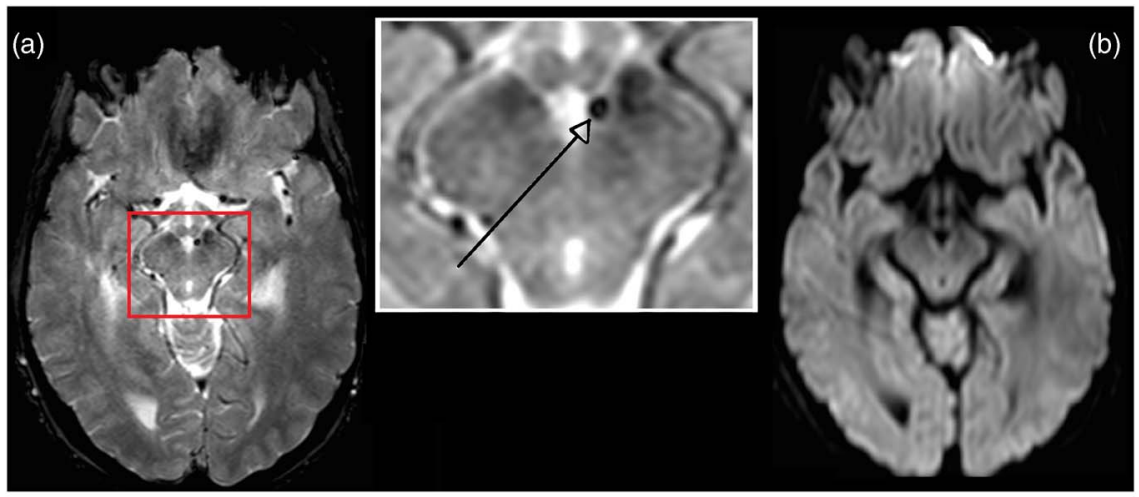

(c)

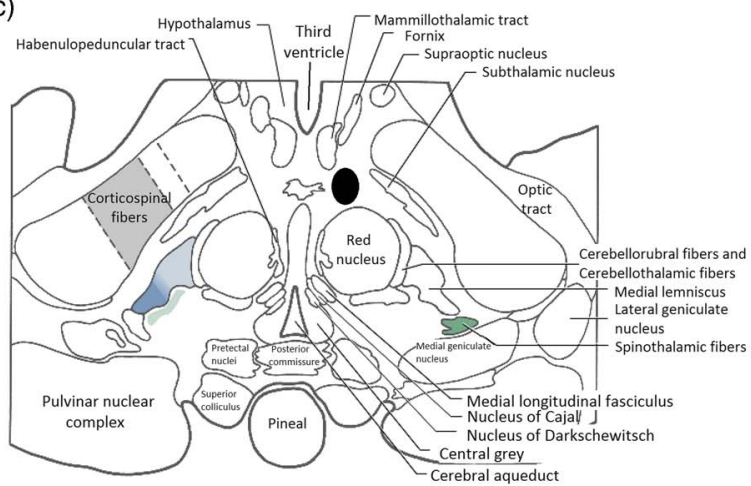

Figure 1: (a) Patient's early post-onset MRI-brain axial GRE $T_{2}$ sequence showing a punctate hypointense lesion (consistent with a CMB) strategically located in the left rostral midbrain, between the red nucleus and the third nerve rootlets (arrow). (b) Corresponding MRI-brain axial DWI (b $\left.1000 \mathrm{~s} / \mathrm{mm}^{2}\right)$ sequence showing no areas of restricted diffusion to suggest acute ischemia in the rostral midbrain. (c) Slightly oblique section through the midbraindiencephalon junction schematically highlighting the anatomic reference structures. The black spot corresponds to the region presumably lesioned by the CMB.

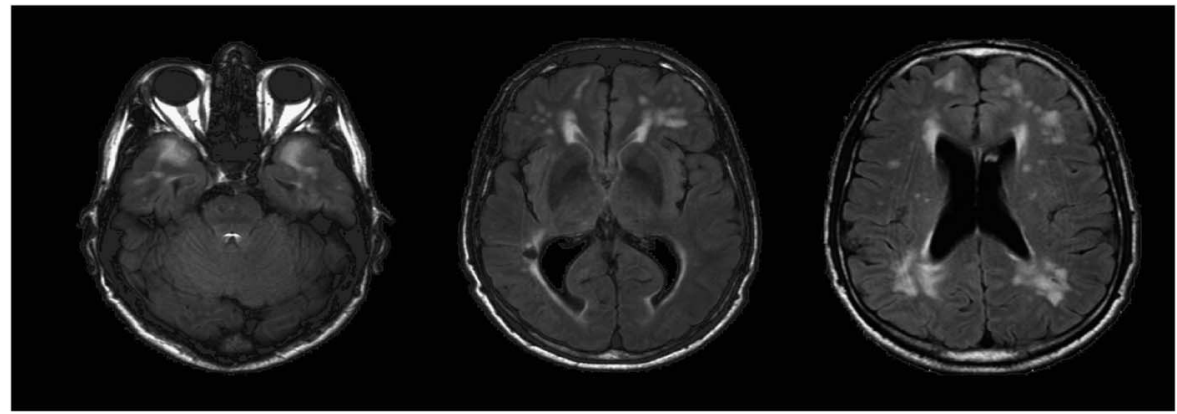

Figure 2: Patient's selected fluid attenuated inversion recovery sequence axial slices showing classical radiological findings of CADASIL including prominent subcortical temporal pole leukoencephalopathy.

in the current case, follow-up high-resolution MRI acquisition performed six months later did not reveal an infarct in this location. Finally, the presumed symptomatic CMB is not exactly centered on the left Interstitial Nucleus of Cajal (INC), as generally expected in cases of right OTR. However, a case has been described of a patient with torsional nystagmus following a lesion in the ventral mesencephalic region between the red nucleus and the third nerve rootlets but without involvement of the INC. ${ }^{8}$

While acknowledging these limitations, this case supports the notion that $\mathrm{CMBs}$ can occasionally cause acute focal neurological deficits. Most likely, neurological dysfunction might be due to some tiny tissue necrosis surrounding the symptomatic CMB, strategically located in a densely packed area such as the brainstem. Possible alternative mechanisms include focal seizure-like activity or migraine aura-like cortical spreading depression, as hypothesized in a few studies reporting characteristic transient focal neurological episodes in cortical CMBs associated with cerebral amyloid angiopathy. ${ }^{9}$ This observation suggests a novel potential non-ischemic mechanism underlying the clinical spectrum of CADASIL disease. 


\section{Statement OF AUTHORShIP}

PV conceived of the idea for the manuscript, conducted the literature review and wrote the manuscript. DB provided details regarding the neuro-ophthalmogical examination and reviewed the manuscript. ND made the initial clinical observation in the patient and reviewed the manuscript. LGL provided guidance regarding interpretation of brain imaging and reviewed and edited the manuscript. AYP conceived of the idea for the manuscript, reviewed the literature, reviewed and edited the manuscript and supervised PV.

\section{REFERENCES}

1. Loitfelder M, Seiler S, Schwingenschuh P, Schmidt R. Cerebral microbleeds: a review. Panminerva Med. 2012;54:149-60.

2. Shoamanesh A, Kwok CS, Benavente O. Cerebral microbleeds: histopathological correlation of neuroimaging. Cerebrovasc Dis. 2011;32:528-34.

3. Teo JTH, Ramadan H, Gregoire SM, et al. Can cerebral microbleeds cause an acute stroke syndrome? Neurol Clin Pract. 2011;1:75-7.
4. Choi JC, Kang SY, Kang JH, Park JK. Intracerebral hemorrhages in CADASIL. Neurology. 2006;67:2042-4.

5. Benameur K, Bykowski JL, Luby M, Warach S, Latour LL. Higher prevalence of cortical lesions observed in patients with acute stroke using high-resolution diffusion-weighted imaging. AJNR Am J Neuroradiol. 2006;27:1987-9.

6. Sylaja PN, Coutts SB, Krol A, Hill MD, Demchuk AM. When to expect negative diffusion-weighted images in stroke and transient ischemic attack. Stroke. 2008;39:1898-900.

7. Hotter B, Kufner A, Malzahn U, Hohenhaus M, Jungehülsing GJ, Fiebach JB. Validity of negative high-resolution diffusionweighted imaging in transient acute cerebrovascular events. Stroke. 2013;44:2598-600.

8. Helmchen C, Rambold H, Kempermann U, Buttner-Ennever JA, Buttner U. Localizing value of torsional nystagmus in small midbrain lesions. Neurology. 2002;59:1956-64.

9. Charidimou A, Peeters A, Fox Z, et al. Spectrum of transient focal neurological episodes in cerebral amyloid angiopathy: multicentre magnetic resonance imaging cohort study and meta-analysis. Stroke. 2012;43:2324-30. 